Revue de droit comparé du travail et de la sécurité sociale

\title{
Le régime des retraites en Italie : la réforme permanente
}

\section{Sylvain Giovanni Nadalet}

\section{(2) OpenEdition}

1 Journals

Édition électronique

URL : https://journals.openedition.org/rdctss/1219

DOI : $10.4000 /$ rdctss. 1219

ISSN : 2262-9815

Éditeur

Centre de droit comparé du travail et de la sécurité sociale

\section{Édition imprimée}

Date de publication : 1 avril 2020

Pagination : 128-141

ISSN : 2117-4350

\section{Référence électronique}

Sylvain Giovanni Nadalet, «Le régime des retraites en Italie : la réforme permanente ", Revue de droit comparé du travail et de la sécurité sociale [En ligne], 1 | 2020, mis en ligne le 01 novembre 2021, consulté le 11 novembre 2021. URL : http://journals.openedition.org/rdctss/1219 ; DOI : https:// doi.org/10.4000/rdctss. 1219

\section{cc)}

Revue de droit comparé du travail et de la sécurité sociale est mise à disposition selon les termes de la Licence Creative Commons Attribution - Pas d'Utilisation Commerciale - Pas de Modification 4.0 International. 


\section{LE RÉGIME DES RETRAITES EN ITALIE : LA RÉFORME PERMANENTE*}

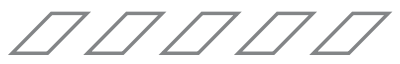

\section{RÉSUMÉ}

La réforme du régime des retraites de 2011 poursuit les réformes entamées dans les années 90, notamment sur le plan du calcul des pensions. Elle introduit toutefois pour l'accès à la retraite un paramètre essentiel qui est celui de l'espérance de vie. Cette volonté d'harmoniser et de rationaliser le régime des retraites s'est cependant heurté par la suite à l'introduction de nombreuses dérogations. L'article met l'accent sur les critères hétérogènes à la base de ces dérogations et s'interroge de manière critique sur le paramètre de l'espérance de vie, en se demandant si celui-ci ne recèle pas des inégalités entre les travailleurs.

MOTS CLÉS : Italie, Retraite, Vieillesse, Sécurité sociale.

\section{ABSTRACT}

The retirement rules reform of 2011 continues the reforms effort started in the 1990 s, particularly in the pension calculation. It also introduces an essential parameter for access to retirement, that of life expectancy. The determination to harmonize and rationalize the pension system was however subsequently prevented by the introduction of numerous exemptions. The essay emphasizes the heterogeneous criteria underlying these derogations and critically questions the parameter of life expectancy, wondering if it produces inequalities between workers.

KEYWORDS : Italy, Pension, Old Age, Social Security.

* Contribution liée à l'activité de recherche du groupe Invecchiamento della popolazione e passaggi generazionali dans le cadre du Projet d'excellence du Département des Sciences Juridiques de I'Université de Vérone, intitulé « Cambiamenti e Tecnologie ». 
e titre de cette contribution pourrait, à première vue, sembler discutable. Face aux interventions incessantes du législateur depuis 20 ans, il devient difficile de distinguer ce qui relève d'une réforme de ce qui est le fait de la conjoncture ou du débat politique du moment. Le terme de réforme dans le langage courant et journalistique est empreint d'ambiguïté, d'aucuns présentant chaque intervention législative (souvent en fin d'année lors du vote du Budget) comme une nouvelle réforme.

Cependant, si l'on s'en tient au strict contenu, ou tout au moins aux finalités annoncées et aux effets escomptés, il est possible d'isoler des périodes de discontinuité majeure dans l'histoire législative italienne, lorsque les interventions du législateur ont eu un impact structurel sur les deux éléments clés du régime des retraites, à savoir les conditions d'accès aux retraites et le mode de calcul des pensions. Le financement par répartition est quant à lui un élément stable du régime de base depuis 1969 (loi n¹53), la capitalisation étant réservée au deuxième pilier à caractère facultatif1.

S'agissant du premier pilier, public et obligatoire, et notamment des règles concernant le régime général ${ }^{2}$, on peut séparer sur un plan chronologique ce qui relève d'un processus unique de réforme - amorcé dans les années 90 et culminant en 2011 du fait de la crise et de la pression européenne - de ce qui relève des dispositifs dérogatoires, qui ont certes toujours existé (les fameuses « normes de sauvegarde ») mais se sont multipliés depuis 2011.

En dépit de ces périodes de discontinuité, il est incontestable que les réformes des années 90, comme celle de 2011 du Gouvernement Monti-Fornero (loi n²14/2011), dessinent au final un parcours de réforme cohérent du point de vue des finalités poursuivies, à savoir retarder les départs à la retraite et rendre les

1 Ce que l'on appelle la prévoyance complémentaire fait néanmoins l'objet d'un cadre juridique strict, établi par le Décret-loi n²52/2005.

2 Les réformes des années 90 ont amorcé un processus d'harmonisation cherchant à aplanir les différences entre régimes spéciaux et le régime général de I'INPS (Institut National de Prévoyance Sociale). Les fonds spéciaux, notamment du secteur paraétatique (électricité, transports, etc.) ont ainsi été supprimés au profit de gestions spéciales de I'INPS qui conservent une autonomie du point de vue comptable mais dont les régimes ont tendance à rejoindre le régime général, notamment pour les nouvelles générations de travailleurs. Les travailleurs les plus probablement épargnés par ce processus d'harmonisation, et aussi par la réforme de 2011, sont ceux des forces de l'ordre, des militaires et des pompiers qui peuvent partir à la retraite sur la base des annuités appliquées avant cette réforme. Depuis 2011, la majeure partie des salariés du secteur public sont eux aussi gérés par I'INPS. Quant aux professions libérales, elles cotisent à des caisses privées qui disposent d'une plus grande autonomie. 
modalités de calcul moins coûteuses (I). Néanmoins, les régimes dérogatoires qui se sont succédés ont rendu le système actuel plus confus, posant ainsi la question de la cohérence générale du régime des retraites au regard de la Constitution (II).

\section{I - LE PROCESSUS DE RÉFORMES DE 1995 À 2011}

Même si elles diffèrent partiellement sur le plan des dispositifs utilisés, les deux lois qui ont remodelé le système italien des retraites hérité des années 60 poursuivent un objectif commun. Sur le mode de calcul, la continuité est plus évidente.

Le mode contributif instauré en 1995 a en effet été généralisé à tous les salariés en 2012, anticipant la fin de la période transitoire et remettant en cause ce que, dans le vocable journalistique français, on nommerait la « clause du grand-père » (A).

En revanche, sur le plan des conditions d'accès à la retraite, l'objectif visant au recul des départs à la retraite s'est effectué non seulement sur la base des structures existantes, mais aussi en tenant compte d'un correctif essentiel, à savoir la prise en compte de l'espérance de vie (B).

\section{A - LA GÉNÉRALISATION DU MODE CONTRIBUTIF OU LA FIN DE LA « CLAUSE DU GRAND-PÈRE »}

Dès les années 90, la nécessité de rendre les pensions moins coûteuses pour les finances publiques a conduit le législateur italien à intervenir sur la définition du salaire moyen et sur le mode de calcul, en liant étroitement le montant des pensions aux cotisations versées. Néanmoins, dans la mesure où ces modifications impactaient le plus souvent défavorablement le montant des pensions, il a donc procédé de manière graduelle et progressive.

Àcet égard une précision s'impose quant à la possibilité pour le législateur italien de modifier les conditions d'accès au régime des retraites. Le fait de prévoir une longue période transitoire ne représente en rien une obligation pour le législateur. Même si la Cour constitutionnelle a reconnu la nécessité de prendre en compte "les expectatives » des travailleurs proches de la retraite lors de l'introduction de nouvelles règles, le fait que ce salarié ait procédé au versement de cotisations ne lui confère pas un "droit » au maintien des règles qui étaient en vigueur durant sa carrière. Bien que le législateur de 1995 ait alors invoqué, pour justifier le caractère transitoire de la réforme, la nécessité de préserver des "droits acquis ", il s'agissait là d'une affirmation de caractère politique ${ }^{3}$. Le véritable droit à la pension ne "s'acquiert » que lorsque le travailleur a rempli les conditions requises sur la base

3 Des limites s'imposent toutefois à l'action du législateur, en vertu des principes de rationalité et de " prudence » des interventions législatives, et du droit à la sécurité juridique. Voir M. D'Onghia, «Retroattività e diritti previdenziali fra (presunta) Intangibilità dei cd. diritti quesiti e solidarietà intergenerazionale », Rivista del Diritto della Sicurezza Sociale, 2019, p. 275. 
des lois en vigueur au moment où il fait sa demande 4 . Par la suite, la " pension» entre dans son patrimoine juridique, elle peut augmenter par l'effet du mécanisme de revalorisation lié à l'inflation, et le législateur ne peut modifier sa base de calcul, bien qu'il puisse appliquer aux retraités les plus riches des cotisations de solidarité 5 .

La nécessité de rendre les pensions moins coûteuses pour les finances publiques était déjà bien présente à l'esprit du législateur lors de la Réforme Amato (d.lgs n503) de 1992, qui a augmenté la base de calcul des pensions selon le mode retributivo fondé - comme le laisse entendre le vocable italien - sur la rétribution perçue et préservée en quelque sorte par le système. Ainsi ce calcul consistait-il à reconnaître au moment du départ à la retraite un pourcentage du salaire, selon la formule de $2 \%$ pour chaque année de travail, ce qui favorisait les carrières longues. C'est donc très progressivement (produisant ses pleins effets seulement en 2001) et en fonction de l'ancienneté des travailleurs que la réforme de 1992 s'est ainsi attachée à élargir à toute la carrière la période prise en compte pour établir le salaire moyen.

La grande réforme de 1995 (loi n³35 également dénommée « Dini-Treu ») fut en revanche de plus ample portée puisqu'elle a introduit un nouveau mode de calcul dit " contributif », se substituant à l'ancien mais destiné à coexister un certain laps de temps avec ce dernier.

Ce mode de calcul crée une sorte de capitalisation fictive à travers laquelle, sur un plan comptable, les cotisations «accumulées » au cours des années et réévaluées, notamment en fonction du PIB, seront transformées en pension par l'application d'un coefficient de transformation (en rente), différent selon l'âge et donc fonction de l'espérance de vie. Ce mode de calcul confère une grande flexibilité à l'Etat dans la mesure où il permet de varier les prestations en fonction des conditions financières de la caisse de retraite. II est d'autant plus souple que le coefficient de transformation est revu tous les 2 ans par arrêté ministériel - après consultation notamment des syndicats ${ }^{6}$ - et abaissé en fonction de l'augmentation de l'espérance de vie, ce qui conduit inévitablement à des pensions moindres le jour où les nouveaux coefficients entrent en vigueur. En outre, il peut se traduire par une baisse des pensions de l'ordre 30\% par rapport aux précédents modes de calcul (à moins d'avoir commencé avec un salaire très haut) et surtout génère un renversement de la logique par rapport aux objectifs de la Constitution en la matière, favorisant les individus sur la base de leur salaire et non selon leurs besoins ${ }^{7}$.

En 1995, il avait été toutefois décidé que ce nouveau système ne s'appliquerait que pour l'avenir, c'est-à-dire pour les périodes de travail consécutives à son entrée en vigueur. Ceci impliquait que les nouveaux entrants sur le marché du travail au $1^{\text {er }}$

4 En Italie, la retraite n'est pas automatiquement versée à l'âge prévu ; elle doit faire l'objet d'une demande du salarié. Aussi, l'annonce de réformes peut-elle générer un recours massif à la retraite pour les salariés encore en activité qui avaient décidé de continuer à travailler afin d'augmenter leur niveau de pensions. Dans le secteur public, l'administration peut licencier d'office si les conditions pour avoir accès à la retraite sont réunies.

5 Décision de la Cour constitutionnelle n¹73/2016.

6 Art. 1 §11, loi n³35 de 1995.

7 Notammentsil'on considèrel'augmentationdumontantrequisparles dernièresréformes. Voir A. Avio, "La vecchiaia della pensione?», Lavoro e diritto, 2013, p. 403. 
janvier 1996 se voient directement appliquer ce mode de calcul, étendu aussi aux travailleurs ayant moins de 18 ans de cotisations à cette date, mais seulement pour la période de travail consécutive selon la formule du prorata (deux modes de calculs pour chaque période). En revanche, les travailleurs ayant plus de 18 ans d'ancienneté au 31 décembre 1995 avaient été épargnés par l'application du nouveau mode de calcul, de sorte qu'ils auraient pu continuer encore quelques années à bénéficier $d u$ système de calcul plus avantageux.

À l'évidence, les effets de la crise sur la population cotisante, l'allongement de la durée de vie, mais aussi et surtout la lettre de la BCE du 5 août $2011^{8}$, ont contraint la Loi Monti-Fornero à mettre fin à l'exemption et à appliquer le mode de calcul contributif au prorata des périodes de travail à partir du $1^{\text {er }}$ janvier 2012 (date d'entrée en vigueur de la loi n²14/2011).

Par la même occasion, il faut souligner que les coefficients de transformation ont été modifiés et portés jusqu'à 70 ans, de manière à inciter les salariés à retarder l'âge de départ à la retraite sur la base des nouvelles règles concernant les conditions d'accès.

\section{B - LES CONDITIONS D'ACCÈS SELON LE « DOUBLE CANAL »}

Si le mode de calcul constitue le fil conducteur entre les deux réformes, les mesures visant à repousser les départs à la retraite via ces conditions d'accès diffèrent en partie. Pour bien en saisir les enjeux, il faut comprendre que l'accès à la retraite fonctionne depuis les années 60 selon un « double canal ». Il est en effet possible d'accéder à la retraite en fonction de son âge à travers la « pension de vieillesse » normale, ou en fonction de la durée de cotisation grâce à la pension dite "d'ancienneté », supprimée en 2012. Ces dernières étaient considérées par la Cour constitutionnelle comme une forme de retraite autonome ${ }^{9}$, et non comme une modalité de départ anticipé.

A l'origine, ces pensions "d'ancienneté » permettaient de partir à la retraite après 35 annuités de travail, indépendamment de l'âge. Néanmoins, les réformes des années 1990 et 2000 ont souvent cherché, par divers ajustements, à empêcher la possibilité pour les salariés concernés d'en bénéficier, ceci en raison du coût engendré. A défaut d'avoir une longue carrière, le salarié (encore majoritairement aujourd'hui une "salariée»), peut accéder à la pension de vieillesse la plus commune, basée sur une durée minimale requise de 20 ans quant à l'ancienneté, ce qui implique naturellement des pensions bien inférieures ${ }^{10}$.

Les réformes ont donc conduit à agir parallèlement sur chacun des régimes d'accès à la retraite, en établissant toutefois des conditions d'accès sensiblement

8 Lettre à l'origine de la réforme bien qu'elle soit dépourvue de toute valeur normative : P. Sandulli, « II sistema pensionistico tra una manovra e l'altra. Prime riflessioni sulla legge $\mathrm{n}$. 2014/2011», Rivista di diritto della sicurezza sociale, 2012, p.1.

9 Cost. 194/1991 et Cost. 73/1992.

10 Que l'Etat toutefois intègre pour atteindre un "minimum » de pensions depuis la loi n¹339/1962, sauf lorsque qu'il s'agit des sujets inscrits après 1995 et dont la pension est intégralement calculée selon le mode contributif. 
plus restrictives pour les personnes entrées sur le marché du travail après 1995 qui, comme évoqué ci-avant, voient leur prestation intégralement calculée selon le mode contributif.

S'agissant des pensions d'ancienneté, le législateur de 1995 avait associé aux 35 annuités la condition d'un âge minimal de 57 ans, et avait porté à 40 ans le nombre d'années de cotisations requises pour accéder à la retraite indépendamment de l'âge. Face à la volonté de la réforme Maroni (loi n²43/2004) de faire passer, dès 2008, le dipôle " annuités-âge » de 35-57 ans à 35-60 ans, le législateur est intervenu in extremis, sous l'effet d'un nouveau changement de gouvernement, avec la loi $n^{\circ} 247 / 2007$. Ce texte permettait d'assouplir l'augmentation grâce à un mécanisme (plus tard dénommé " quotas ») intégrant la somme de l'âge et celle de l'ancienneté, et dont le montant (" quota 93 », " quota 94 », etc.) a augmenté graduellement jusqu'à arriver au seuil de la réforme de 2011 au « quota $95 »^{11}$.

La doctrine a pu s'interroger sur le sens d'un tel critère quantitatif comme base du droit à la retraite (voir infra §II, B). Mais de toute évidence il n'a d'autre fonction dans l'esprit du législateur que de fournir une variable d'ajustement permettant d'adapter graduellement les départs à la retraite aux exigences de l'équilibre financier des retraites.

L'un des principaux objectifs de la réforme Monti-Fornero fut d'abolir la pension d'ancienneté et de la remplacer par une pension dite « anticipée », qui revient à l'esprit initial d'un droit à la retraite indépendamment de l'âge, mais pour des carrières très longues puisque le nombre d'annuités requises est passé en 2012 à 41 ans pour les femmes et 42 ans pour les hommes. Or comme pour les pensions de vieillesse, la durée de cotisation requise augmentera parallèlement à l'espérance de vie (voir ci-après). Il convient également de signaler que la loi, en plus d'introduire des mécanismes d'incitation économique par le biais de coefficients de transformation, avait aussi prévu des pénalités financières - se traduisant par une réduction mesurée en pourcentage de la pension ${ }^{12}$ - à l'égard des travailleurs accédant aux pensions anticipées avant l'âge de 62 ans, créant de fait une sorte d'«âge pivot ». L'entrée en vigueur de cette pénalité, discutable notamment en raison des limites appliquées aux périodes couvertes par les cotisations, a toutefois été plusieurs fois repoussée avant d'être définitivement abandonnée.

Sur le front des pensions de vieillesse, la Réforme Amato de 1992 avait amorcé une augmentation très progressive de l'âge minimum, différenciée selon le genre (de 55 à 60 ans pour les femmes et de 60 à 65 ans pour les hommes) également assortie d'une augmentation du nombre d'années de cotisation (de 15 à 20 ans). Par la suite, et en dépit du fait que le mode de calcul contributif était appliqué pour les nouveaux entrants sur le marché du travail, la réforme Dini-Treu de 1995 a lié, de manière cohérente et indissoluble, ce mode de calcul aux conditions de retraites.

Prévoyant même à l'origine un âge d'accès à la retraite flexible " au choix » (entre 57 et 65 ans) pour ces nouveaux travailleurs, ces derniers étaient soumis à

11 Ce qui impliquait de remplir la condition cumulative de 60 ans et 35 ans de cotisations, les 40 années de cotisations indépendamment de l'âge restant pour leur part inchangées.

12 Il était prévu un abaissement de la pension de 1\% pour le salarié partant à 61 ans et de $2 \%$ pour chaque année antérieure. 
un régime spécifique appelé " pension de vieillesse contributive ", associant aux conditions d'âge et d'ancienneté prévues pour les travailleurs plus anciens une condition supplémentaire - qui deviendra la condition principale -, selon laquelle au moment de la demande, le calcul du montant de la pension donne droit à un montant dépassant d'un certain pourcentage (20\%) le minimum vieillesse (revenu d'assistance) $)^{13}$.

Si telle était la configuration du cadre juridique au moment de la crise de 2008, dès 2009 et jusqu'en 2011, différentes mesures adoptées par décrets-lois ${ }^{14}$, avant même l'adoption de la réforme Monti-Fornero, ont jeté les bases des aspects les plus novateurs et « perturbants » du régime actuel.

Le principe consiste à lier automatiquement l'âge de la retraite à l'espérance de vie. Ce mécanisme de report automatique triennal, initialement prévu pour s'appliquer en $2015^{15}$ mais anticipé pour $2013^{16}$, a finalement été repris par la réforme de 2011 qui l'a rendu biennal à partir de 2012. Ce même mécanisme de report automatique s'applique aux annuités nécessaires pour les pensions anticipées, pour l'octroi desquelles les travailleurs ont vu les annuités requises augmenter progressivement jusqu'à parvenir en 2019 à 41 ans et 10 mois pour les femmes, 42 ans et 10 mois pour les hommes.

Le système de l'adaptation automatique a été modifié par la loi de finances $2018^{17}$ pour faire en sorte que l'adaptation automatique ne puisse dépasser une durée de 3 mois. Par ailleurs, il faut noter qu'une éventuelle diminution de l'espérance de vie ne provoque pas une diminution des critères mais permet d'éviter - ou de tempérer - une possible augmentation future. Ceci a conduit, par un décret du 5 novembre 2019, à bloquer l'âge de la retraite à 67 ans jusqu'en 2022 (les analyses statistiques ayant révélé une augmentation de l'espérance de vie de moins d'un mois). Par le même acte, il a été décidé de " geler » jusqu'en 2026 la durée de cotisations nécessaire indiquée plus haut pour l'accès à la pension anticipée.

Le second mouvement engagé avant la réforme Monti-Fornero, accéléré par cette dernière, a consisté à prévoir pour le régime général une harmonisation de l'âge légal de la retraite entre hommes et femmes, à l'instar de ce qui s'était produit pour les salariés du secteur public suite à un arrêt de la CJUE de novembre $2008^{18}$. Initialement prévu pour 2028, le rapprochement des âges de la retraite,

13 Par la suite, la réforme Maroni de 2004 abolira l'âge flexible et conduira à ce que les jeunes générations se voient appliquer les conditions d'âge fixe, prévues pour les pensions de vieillesse et la pension d'ancienneté, mais toujours avec la condition ultérieure de lier l'accès à la retraite au montant des pensions.

14 Dispositifs en principe liés à l'urgence, par la suite convertis en lois par le Parlement.

15 Il s'agit notamment des D.L. n 78/2009, n 78/2010 et n²01/2011 (convertis respectivement en lois $n^{\circ} 102 / 2009, n^{\circ} 122 / 2010$ et $\left.n^{\circ} 124 / 2011\right)$.

16 D.L. 98/2011 (converti en loi n¹11/2011).

17 Loi n²05/2017, art. 1, al. 146, (modifiant l'art. 24, al. 13, D.L. 101/2011 converti en loi $n^{\circ} 214 / 2011$ ). Dès 2021, l'espérance de vie sera mesurée tous les 2 ans en comparant les 2 dernières années avec les 2 années précédentes.

18 Arrêt de la CJUE du 13 novembre 2008 (C. 46/2007). Sur ce sujet, voir également P. Bozzao, «La Corte di giustizia coglie nel segno sbagliando a mira: paradossi del sistema previdenziale del pubblico impiego », Diritti, Lavori, Mercati, 2009, p. 147. 
parallèlement à son allongement consécutif à l'espérance de vie, a été réalisé en 2018 suite à la réforme Monti-Fornero ; il est aujourd'hui unifié à l'âge de 67 ans.

Le dernier aspect structurel général sur lequel la réforme Monti-Fornero n'est pas revenue, si ce n'est pour le rendre plus restrictif, est de subordonner l'accès à la retraite pour les travailleurs dont la pension est calculée exclusivement selon le mode contributif (car entrés sur le marché du travail depuis 1996) au fait que leur pension ne soit pas trop basse.

La réforme Monti-Fornero avait annoncé que ces derniers ne relèveraient plus du régime spécifique étudié ci-dessus, et qu'ils pourront partir à la retraite selon les règles fixées pour les pensions de vieillesse ou anticipée. En réalité et comme par le passé, une condition ultérieure continue à s'imposer dans leur cas pour partir à la retraite, puisque le calcul du montant de leur pension doit dépasser encore plus largement le minimum vieillesse, à moins d'atteindre l'âge fatidique de 70 ans, seuil qui toutefois augmentera aussi en fonction de l'espérance de vie ${ }^{19} \ldots$

Enfin, parmi les changements à mettre au bénéfice de la Réforme-Fornero et à placer sous le signe des mesures rationnelles, il faut citer l'abandon du mécanisme dit des "fenêtres ", consistant à octroyer la pension non pas immédiatement - une fois remplies les conditions d'ancienneté ou de vieillesse - mais passé un certain délai administratif/comptable, qui s'est au final transformé ${ }^{20}$ en une façon détournée de retarder l'exercice effectif du droit à la pension ${ }^{21}$.

\section{II - LA DIVERSIFICATION DES RÉGIMES DÉROGATOIRES ET LA QUESTION DE LA COHÉRENCE GÉNÉRALE DU RÉGIME DES RETRAITES}

C'est dans un souci d'équité, notamment vis-à-vis des jeunes générations, que la réforme de 2011 annonçait comme objectif « la suppression des privilèges et l'adoption de clauses dérogatoires seulement pour les catégories les plus défavorisées ». Ainsi, à peine mise sur les rails la loi prévoyait des dérogations pour

19 Ainsi la pension de vieillesse, qui requiert au moins 20 ans de cotisations, ne pourra être perçue qu'à condition de percevoir un traitement supérieur de $50 \%$ au minimum vieillesse, ou alors d'attendre d'avoir aujourd'hui 71 ans. On retrouve le même principe dans les règles relatives à la pension anticipée énonçant (de manière absolument incohérente avec l'idée d'une retraite basée exclusivement sur la durée de cotisation) que les travailleurs au régime contributif, qui probablement auront du mal à réunir le nombre d'années requises, pourront demander leur retraite à 63 ans avec seulement 20 ans de cotisation, à condition toutefois que la pension atteigne un montant correspondant à 2,7 fois le minimum vieillesse. II est par ailleurs précisé que les 20 ans de cotisation ne prendront pas en compte les périodes où le salarié a bénéficié d'une couverture financée par l'Etat (chômage, maladie, etc.).

20 Initialement, il s'agissait de périodes déterminées, fixées dans l'année. Face à l'urgence de la crise et pour repousser l'échéance des retraites, la loi de 2010 a introduit la fenêtre flexible qui consiste en une période allant de 12 à 18 mois selon les circonstances.

21 Même si le salarié, en poursuivant son travail durant cette période, continue d'alimenter son montant contributif individuel qui servira pour la partie de pension calculée selon le mode contributif. 
les salariés de certaines classes d'âge qui auraient dû subitement être obligés de travailler 5 ans de plus ${ }^{22}$, ou ceux concernés au moment de l'entrée en vigueur par des procédures de réduction de personnel, ce qui était somme toute compréhensible.

Sous l'égide des gouvernements successifs, c'est bien souvent à travers les lois de finances de fin d'année et donc sous l'effet des pressions politiques, que se sont multipliées les dérogations dans lesquelles ont resurgi des critères empruntés à la législation précédente ; ceci au point de rendre vaine l'intention d'harmoniser et de clarifier cette réforme, pour à l'inverse faire du régime des retraites un chantier permanent défini comme « la jungle » des retraites » ${ }^{23}(\mathbf{A})$.

La diversité des motifs qui apparaissent dans ces correctifs, comme dans les tentatives de contre-réformes à caractère transitoire, complexifient le régime des retraites dont on peine à trouver une cohérence d'ensemble, notamment au regard des exigences de protection fixées originellement par la Constitution (B).

\section{A - L'ÉVENTAIL DES DISPOSITIFS DÉROGATOIRES : POUR QUELS BÉNÉFICIAIRES?}

Cherchant à éviter l'effet d'inventaire auquel pourrait contraindre la forte inventivité du législateur italien, il est important de relever, au sein de chaque régime dérogatoire, les raisons qui ont conduit aux privilèges accordés à certains travailleurs, afin de distinguer celles qui ont un fil conducteur de celles qui s'en détachent.

Une première catégorie de dispositifs dérogatoire a consisté à prendre en compte ce qui peut être qualifié dans le vocable juridique français de "pénibilité ", mais qui a donné lieu à une plus grande variété linguistique et conceptuelle dans la législation italienne. Avant même la loi de 2011, un décret-loi (n67/2011) avait créé une catégorie de travaux particulièrement " usants ${ }^{24}$, allégeant de 3 ans les conditions d'accès à la retraite alors en vigueur. La loi Monti-Fornero a remplacé cet avantage par l'attribution de "quotas » spécifiques (selon les mécanismes indiqués ci-avant) prenant notamment en compte la quantité de travail nocturne.

La seconde intervention du législateur a consisté à créer une catégorie plus large de travaux qualifiés de " pénibles " (gravosi) pour y associer différents avantages ${ }^{25}$ au profit des travailleurs concernés : les soustraire au recul automatique

22 Ceux de la classe d'âge " 1952 ». Ainsi, l'art. 24 al.15 bis du D.L. n²01/2011 a prévu un régime dérogatoire basé sur une hausse graduelle différenciée.

23 M. Cinelli et C.A. Nicolini, « Verso la fine della XVII legislatura. La manovra finanziaria per il 2018. Ancora pensioni, sgravi per le assunzioni dei giovani... e non solo ", Riv. It. Dir. Lav., 2018, III, p. 57.

24 Ainsi sont-ils définis de manière consensuelle et pour les distinguer d'autres catégories, même si le texte parle de travaux particulièrement « fatigants et lourds ». Voir sur ce thème S. G. Nadalet, "L'attuazione della delega sui lavori usuranti: chi lo dice che si vive (solo) tre anni di meno?", Lavoro e Diritto, 2011, p. 557.

25 Comme le remarquent M. Cinelli et C.A. Nicolini (op. cit., p. 62), on décompte au total 4 catégories de travaux auxquelles on associe des effets juridiques différents. 
du départ à la retraite lié l'espérance de vie ${ }^{26}$, mais aussi les rendre bénéficiaires des deux dispositifs dérogatoires.

Le premier avantage, introduit de manière stable par la loi de finances $2017^{27}$, permet aux salariés ayant commencé à travailler jeunes dits "précoces", d'anticiper d'une année l'accès à la retraite par rapport à la retraite déjà anticipée ${ }^{28}$ sous réserve qu'ils remplissent certaines conditions. Si le fait d'avoir été rattaché à l'une des 15 catégories de travaux pénibles de façon continue ${ }^{29}$ est une des conditions complémentaires prévues pour que ces travailleurs puissent être admis au bénéfice prévu, ce n'est en revanche pas la seule.

Alternativement, il est également possible de faire valoir le fait d'être un chômeur sans indemnité chômage depuis au moins 3 mois, de prendre soin d'un parent en situation de handicap grave depuis au moins 6 mois, ou encore d'avoir une incapacité de travail d'au moins $74 \%$.

On retrouve la même hétérogénéité dans les exigences prises en compte pour accéder au second dispositif d'anticipation, cette fois-ci de nature expérimentale, appelé APE, introduit lui aussi en 2017 pour favoriser certains travailleurs voulant se retirer à l'âge de 63 ans et à condition de justifier de 30 années de cotisations. II s'agissait d'un système d'anticipation particulièrement complexe à travers lequel le travailleur, qui n'est pas techniquement à la retraite, reçoit un prêt couvrant la période manquante pour arriver à l'âge requis pour la pension de vieillesse, moment à partir duquel il devra rembourser ce prêt par des prélèvements sur sa pension pour une durée allant jusqu'à 20 ans (une assurance vie doit être souscrite).

Bien que ce dispositif expérimental, ouvert à tous, ait eu peu de succès ${ }^{30}$, il a été prévu que le travailleur remplissant les conditions " alternatives » indiquées ci-dessus (travaux pénibles, chômage, assistance aux handicapé, etc.) pouvait voir ce prêt pris en charge par l'Etat. Ce dernier dispositif appelé «APE sociale», a été prorogé pour 2020.

Force est de constater que si l'absence de connexion logique entre les critères justifiant des conditions d'accès privilégié à la retraite peut surprendre (type de travail, situation d'emploi, handicap, etc.), d'autant que s'agissant de dispositifs alimentés par des fonds aux ressources limitées, les travailleurs sont de fait en concurrence entre eux pour y accéder et il ne s'agit pas là d'une nouveauté.

Des critères semblables figuraient déjà durant la phase qui a suivi l'entrée en vigueur de la réforme de 2011, lorsque que fut réalisé tardivement le fait que la disposition exonérant les salariés touchés par des restructurations, qui avaient accepté des primes de départ sur la base de la législation précédente, était insuffisante à couvrir le nombre de salariés concernés. Ainsi a-t-on vu poindre au cours des années suivantes une série de décrets dits de "sauvegarde " destinés

26 Art. 1 al. 147, loi n²05/2017.

27 Art. 1, al. 199-205, loi n²32/2016. Le régime a été élargi par la loi de finance 2018 (loi $n^{\circ} 205 / 2017$, art, 1 al. 162-164 et al. 166).

28 A présent 41 ans (ou « quota 41 »).

29 C'est-à-dire 6 mois au cours des 7 derniers mois.

30 Il a pris fin en 2019. 
à maintenir en vigueur les règles antérieures à la réforme (y compris les fenêtres), sur la base de critères non seulement liés à la nature de l'emploi, mais à aussi à des exigences ayant trait aux charges familiales ${ }^{31}$.

Dans le même ordre d'idée, au-delà des bénéfices spécifiquement liés à la maternité, l'harmonisation de l'âge de la retraite entre hommes et femmes n'a pas empêché le maintien de dérogations particulières, eu égard à la permanence d'un modèle familial qui présuppose que les femmes se consacrent davantage au care et ont accès moins facilement aux pensions basées sur l'ancienneté.

Ceci explique aussi que, depuis son introduction en 2004, ce que l'on appelle "l'option Femme " $^{32}$ ait été prorogée à plusieurs reprises, autorisant les femmes à partir plus tôt à la retraite, mais avec une pension probablement moindre car calculée intégralement selon le mode contributif.

En 2018, à ce panorama déjà fragmenté s'est ajoutée une mesure qui n'obéit pourtant à aucune des exigences de protection précédemment évoquées, mais plutôt à la volonté de supprimer certains effets de la réforme Fornero-Monti, en particulier l'introduction du « quota 100 » (article 14 du décret 4/2019) qui génère la possibilité de partir à la retraite dès 62 ans avec 38 ans de cotisation. La réminiscence du « quota », qui traduit une volonté politique d'un retour en arrière - si elle n'est pas prorogée -, devrait s'éteindre fin 2020, le gouvernement ayant entre temps changé.

Le tableau qui précède ayant trait seulement au régime général, synthétisé en occultant certains particularismes (emploi public) et problèmes juridiques liés à la multiplication des dispositifs, offre actuellement en Italie une douzaine de régimes prévoyant autant de conditions différenciées d'accès à la retraite. Ce choix dépendra de la situation personnelle du travailleur (carrière, rémunération, type de travail) mais aussi du fait que, comme aujourd'hui, toutes les dernières années de travail donnent droit à une partie de la retraite calculée selon le mode contributif : si le salarié contenue à travailler, il augmente sa retraite. Tout accès à un dispositif d'anticipation implique donc, par définition, une pension plus basse.

Enfin, symbole d'un retour en arrière - et aux mauvaises habitudes - les "fenêtres » de 3 mois ont été réintroduites en 2018 afin de retarder de quelques mois le versement des pensions de vieillesse et d'ancienneté.

31 Il avait été nécessaire d'introduire 8 décrets de sauvegarde, prévoyant l'application des règles précédentes non seulement pour les salariés ayant accepté de quitter l'entreprise contre une prime à "l'exode » qui avaient été calculée sur la base des règles précédentes et qui se trouvaient donc sans ressource en vertu des nouvelles règles, mais aussi à des chômeurs de longues durée ou prenant en charge des parents handicapés.

32 Initialement «l'option Femme » permettait de partir à la retraite selon les règles prévues pour la pension d'ancienneté( 35 ans de cotisations et 57 ans). Al'heure actuelle, la condition d'âge a été portée à 58 ans, mais à celle-ci s'ajoute le temps d'attente d'une «fenêtre » de 12 mois (D.L. 4/2019). 


\section{B-RATIONALITÉÉCONOMIQUEOURATIONALITÉPROTECTRICE:QUELLEFONCTION POUR LE CRITÈRE DE L'ESPÉRANCE DE VIE ?}

Si la doctrine a eu raison de souligner que les dispositifs transitoires ou expérimentaux ne peuvent être pris en compte pour évaluer la cohérence du système d'un point de vue général ${ }^{33}$, il faut souligner que non seulement cela ne préjuge en rien de leur durée ${ }^{34}$, mais aussi que l'on voit malgré tout poindre parmi les exigences de protection des éléments récurrents qui s'ajoutent aux questionnements concernant les fondements du droit à la retraite.

La doctrine italienne peine en effet à trouver des éléments cohérents dans l'architecture du système de protection sociale hérité de la Constitution, qui énonce à son article 38 alinéa 2 le droit à une " prestation adaptée aux exigences de vie » en cas de « vieillesse ». II faut dire que l'application de cette disposition est constamment mise en balance avec d'autres principes constitutionnels qui en amoindrissent l'effet, ou orientent différemment son application, d'une part sur le plan de la mesure de la prestation considérée comme "adaptée ", et d'autre part, sur les conditions d'accès à la retraite ${ }^{35}$.

L'affirmation récente du principe de maintien à l'équilibre des finances de l'Etat - aujourd'hui inscrit à l'article 81 de la Constitution - constitue l'un de ceux-là. Mais d'autres principes ou valeurs comme le devoir/droit de travailler pour participer à une société centrée sur la valeur travail, ou d'autres intérêts majeurs comme la lutte contre le travail dissimulé, expliquent qu'en Italie ait été généralisée la possibilité pour le retraité de cumuler la retraite et un nouveau travailib. Ainsi, il est difficile d'identifier le fondement des régimes de retraite uniquement à la lumière de ce que l'article 38 (précité) présente comme une condition de "vieillesse » du travailleur car, dans l'optique italienne, ceci ne correspond pas à un « risque social » mais à une situation de besoin donnant droit à une prestation correspondant à la participation du travailleur à la société ${ }^{37}$.

Dans la législation, on distingue d'ailleurs différents paramètres faisant référence à l'âge, à l'instar de l'âge de 70 ans qui donne à employeur la possibilité de licencier sans justification un salarié dans le secteur privé. La doctrine s'accorde donc pour distinguer l'âge de la retraite - prévu pour la pension de vieillesse - d'un âge de vieillesse biologique correspondant à l'invalidité présumée survenir au seuil des 70 ans.

Cependant, on observe que ce seuil, permettant aux travailleurs affiliés au régime contributif de partir à la retraite indépendamment du montant de leur

33 G. Canavesi, "L'età pensionabile nella prospettiva previdenziale », Variazioni su temi di Diritto del Lavoro, 1/2017, p. 57 : www.dirittolavorovariazioni.com

34 Comme on l'a vu pour l'APE social et pour l'Option Femme, la prorogation des dispositifs expérimentaux est courante en matière de législation sociale.

35 Voir récemment S. Scagliarini, « II bilanciamento tra diritti e risorse finanziarie nella più recente giurisprudenza costituzionale », Rivista del Diritto della Sicurezza Sociale, 2019, p. 331.

36 G. Canavesi, op. cit.

37 Sur la dimension de « rétribution » des prestations de retraite, voir notamment R. Casillo, La pensione di vecchiaia, un diritto in trasformazione, ESI, Napoli, 2016. 
retraite, est d'ores et déjà passé à 71 ans du fait de l'augmentation de l'espérance de vie...

Au-delà des différentes conditions d'âge, à partir desquelles se déclinent diverses conditions de "besoin ", il n'en demeure pas moins que c'est le critère de la seule ancienneté qui, par le passé privilégiant les longues carrières et la participation du travailleur au financement de la prévoyance, a constitué une anomalie ${ }^{38}$. De ce point de vue, on a considéré que l'introduction du système des "quotas » a permis en quelque sorte de transformer la pension d'ancienneté en une prestation plus ou moins liée à la vieillesse.

Aujourd'hui, la question se pose donc de savoir si le retour au seul critère de la durée de cotisation pour la " pension anticipée »- une durée plus élevée toutefois - permet, comme énoncé à l'époque par la Cour constitutionnelle au sujet des pensions d'ancienneté (voir supra $\S l, B$ ), de considérer qu'il s'agit d'une forme de pension spécifique non liée à la vieillesse ${ }^{39}$.

Toutefois, c'est l'automatisme de l'adaptation de l'âge de la retraite à l'espérance de vie, venu se greffer sur ce double canal, qui prête le flanc aux critiques majeures. En impliquant une augmentation de l'âge de départ à la retraite indépendante de la vieillesse, ce mécanisme pourrait conduire, selon une doctrine, à faire du « risque de mort » le paramètre essentiel de l'accès à la retraite, ce qui éloignerait radicalement les prestations de retraite de leur fonction originelle de protection énoncée par l'article 38 de la Constitution ${ }^{40}$.

Cela dit, si l'on accepte qu'à travers le critère de l'espérance de vie l'impératif de l'équilibre financier prime sur les objectifs de protection, alors se pose la question de l'égalité des travailleurs face à un tel paramètre qui, selon certaines études épidémiologiques, n'est pas égal selon les carrières et dépend grandement du type de travail. Le principe d'égalité substantielle (article 3 alinéa 2 de la Constitution) devrait a minima commander de différencier l'âge de départ à la retraite en fonction des écarts quant à l'espérance de vie (présumés, comme l'âge d'invalidité physique $)^{41}$.

38 A. Aurilio, "La pensione di anzianità: alle origini di una anomalia », Riv. dir. sic. soc., 2003, p. 633.

39 R. Pessi, Lezioni di diritto della previdenza sociale, Cedam, 2016, p. 418

40 R. Casillo, «L'attesa di vita nei requisiti di accesso alla pensione: una prospettiva giuridica », in Rivista del Diritto della Sicurezza Sociale, 2016, p. 117.

41 Voir sur ce sujet : notamment pour les références aux études épidémiologiques effectuées à travers la banque de données WHIP (Work History Italian Panel). Voir également sur cette question S. G. Nadalet, "L'attuazione della delega sui lavori usuranti: chi lo dice che si vive (solo) tre anni di meno?», op. cit. La question pourrait se poser de l'inégalité entre hommes et femmes, du point de vue de l'espérance de vie ; R. Casillo, « L'attesa di vita nei requisiti di accesso alla pensione: una prospettiva giuridica ", op. cit., p. 133. L'auteur conteste toutefois fermement la validité juridique et factuelle d'une redistribution des inégalités entre sexes basée sur l'accès à la retraite. 


\section{Conclusion}

A l'heure où sont écrites ses lignes, le Gouvernement italien actuel a annoncé une nouvelle réforme qui s'articulerait autour de la « pénibilité », ou plus exactement du caractère « usant » de certaines professions, laissant ainsi sous-tendre la voie d'une harmonisation des catégories professionnelles considérées comme privilégiées.

Parallèlement se fait jour l'idée d'une pension de « garantie », visant à compenser le faible montant des futures retraites intégralement calculées sur la base du mode contributif. Ceci engendrerait probablement un recours aux ressources typiques de l'assistance pour subventionner les besoins d'une prévoyance qui ne pourrait, de toute évidence, reposer exclusivement sur les cotisations des actifs.

En attendant cette énième grande réforme, l'histoire récente du régime des retraites en Italie a démontré que même si la conformité au cadre constitutionnel retient l'attention des juristes, force est de constater que depuis longtemps, qu'il s'agisse de réformes structurelles ou de contre-réformes aussi épisodiques soientelles, le législateur obéit à des exigences situées parfois aux antipodes de la Constitution.

Pris en tenailles entre les demandes de protection sociale provenant de la société et l'impératif de l'équilibre financier, il a parfois été conduit à adopter des critères d'accès à la retraite faisant office de variables d'ajustement comptable alors que, pour le calcul des retraites, cette fonction est déjà assurée par l'instrument du coefficient de transformation en rente typique du mode contributif. Certains économistes estiment ainsi que le mode de calcul contributif suffirait à réaliser l'équilibre du régime général, sans devoir paramétrer les conditions d'accès à l'espérance de vie.

Peut-être une prise en compte de l'espérance de vie différenciée en fonction des carrières, qui de manière plus ou moins évidente est sous-jacente dans la question de la pénibilité, représente-t-elle un paramètre objectif permettant de rétablir un lien entre ces impératifs et surtout le seul, sur un plan politique, à pouvoir justifier des différences de traitement.

\section{SYLVAIN GIOVANNI NADALET}

Maître de conférences à l'Université de Vérone

\section{Thèmes de recherche : Droit de la sécurité sociale, Travailleurs de la santé.}

\section{Publications:}

S. G. Nadalet, "Sicurezza sociale per i lavoratori poveri e coordinamento delle tutele ", Lavoro e diritto, 2018, n 4, p. 633.

$\sim$ S. G. Nadalet, « L'attuazione della delega sui lavori usuranti: chi lo dice che si vive (solo) tre anni di meno? », Lavoro e diritto, 2011, n 3, p. 557. 